\title{
The role of early-onset-sepsis in the neurodevelopment of very low birth weight infants
}

\author{
Tjark Ortgies ${ }^{*}$, Michael Rullmann², Dorothée Ziegelhöfer ${ }^{1}$, Annett Bläser ${ }^{1}$ and Ulrich H. Thome ${ }^{1}$
}

\begin{abstract}
Aims: The study investigated a putative association between early-onset-sepsis (EOS) and poor neurodevelopmental outcomes at 2 years corrected age in very low birth weight infants.

Methods: This was a single-center cohort study on infants weighing less than $1500 \mathrm{~g}$ with a gestational age below 35 weeks at birth born between 2008 and 2011. Neurodevelopmental outcomes were assessed at follow-up with the Bayley Scales of Infant Development-II. EOS was defined as either culture-proven EOS or clinical EOS using blood culture, CrP levels, and clinical symptoms and treatment. Neurodevelopmental impairment (NDI) was defined as one or more of the following: Mental Developmental Index (MDI) and/or Psychomotor Developmental Index (PDI) scores lower than 70; presence of cerebral palsy.

Results: Of 405 eligible newborns in the study period 166 were included. Two had culture-proven and 29 clinical EOS. Median MDI scores in patients with EOS were 96 (IQR: 86-106) and in the control group 94 (84-106, $p=0.77$ ). PDI scores in patients with EOS were $96(86-106)$ and in the control group 99,5 (92-103, $p=0.03)$. Of infected patients $7 / 31$ (24\%) showed NDI as defined, whereas only 11/135 (8\%) showed NDI in the control group (OR 3.3, $p=0.03$ ). Multiple regression analyses identified chorioamnionitis and poor CRIB-Scores as individual risk factors for $\mathrm{MDI}$ or PDI values $<70$.

Conclusion: In our study, EOS among VLBW-infants significantly impaired the neurodevelopment at 2 years corrected age. As shown in previous reports infection continues to be a problem and strategies for a reduction need further improvement.
\end{abstract}

Keywords: Early-onset-Sepsis, Neurodevelopment, Preterm, VLBW

\section{Introduction}

Very low birth weight infants (VLBW, birth weight < $1500 \mathrm{~g}$ ) are more susceptible to brain injury than term infants. This may lead to adverse long-term neurodevelopmental outcomes [1, 2]. While survival rates of VLBW-infants have increased in recent years, the rates

\footnotetext{
*Correspondence: tjark.ortgies@gmx.de

'Division of Neonatology, University Children's Hospital Leipzig, Liebigstraße 20, 04103 Leipzig, Germany

Full list of author information is available at the end of the article
}

of long-term developmental impairments have not decreased $[3,4]$.

In their review article, Saigal et al. [2] pointed out that about one quarter of surviving preterm infants have substantial neurological morbidity. Common impairments affecting the central nervous system (CNS) are intellectual disability, cerebral palsy, and sensory impairments [1]. Several mechanisms have been proposed as to how systemic inflammation and subsequently elevated cytokine levels in mother and child in the perinatal period may damage brain parenchyma [5-7]. 
Other well established risk factors for adverse longterm neurological outcomes are low gestational age, low birth weight, male gender, bronchopulmonary dysplasia (BPD), intraventricular hemorrhage (IVH), necrotizing enterocolitis (NEC), periventricular leukomalacia (PVL), and retinopathy of prematurity (ROP) $[2,8,9]$.

The aim of our study was to assess the effects of earlyonset-sepsis (EOS) on VLBW infants' neurological development. EOS is usually defined as onset of an infection within the first $72 \mathrm{~h}$ of life in hospitalized infants with a proof of pathogen by blood culture [9-11]. Numbers regarding the incidence of culture-proven EOS in VLBW infants range from 1 to 28 per 1000 live births depending on gestational age, birth weight, ethnicity and intrauterine infection among many other factors $[9,10,12]$.

However, clear sepsis symptoms can occur even though attempts to culture the causative organism may remain unsuccessful $[4,13]$. This situation has been defined as clinical sepsis in similar research $[6,8,10$, 14-16]. Recognizing clinical sepsis can be enhanced by long established blood biomarkers such as C-reactive protein $(\mathrm{CrP})$ and interleukins $[15,17,18]$. The definition of EOS as an infection starting within the first $72 \mathrm{~h}$ of life reflects the proposed pathophysiology, as pathogens are considered to be transmitted vertically from mother to child in the pre- and perinatal period [4, 9, 10]. Pathogens causing EOS are most commonly Group-B-Streptococcus (GBS) and, increasingly, Escherichia coli and several less common bacterial pathogens, but they may also include candida or viral pathogens $[4,9,10,12,19]$.

Few reports have been published regarding a possible negative effect of infection on neurodevelopmental outcomes in premature infants. These reports were predominantly based on infants born in the 1990s and included both entities of neonatal infection (EOS and LOS) $[6,8,20,21]$. The most recent study evaluated infants with either a positive blood culture or prolonged antibiotic treatment, which, however, was solely based on clinical symptoms [11]. According to the above mentioned mechanisms causing damage to brain parenchyma, the immaturity of the CNS is believed to be a substantial factor $[5,7]$. Therefore, in our study we focused on patients with EOS and clinical-EOS using a more rigorous clinical EOS definition, which included mandatory laboratory abnormalities. We hypothesized that clinical as well as culture-proven EOS might be associated with adverse neurodevelopmental outcomes.

\section{Methods}

Patients and data acquisition

The cohort of our retrospective study included all inborn VLBW infants with a gestational age of less than 35 weeks cared for in our tertiary care neonatal intensive care unit (NICU) in the years 2008 through 2011. Data was retrieved from the hospital records of these patients and their mothers from during their stay in our hospital. Patients with congenital defects and syndromes were excluded from our cohort. Equally patients, who were exposed to maternal drug abuse during pregnancy and received postnatal opiate substitution, and those with incomplete hospital records were excluded, as were patients who died.

Only patients who returned for a follow-up visit around the corrected age of 24 months for the neurodevelopmental assessment were included in the final analysis. Basic demographic and clinical data of patients with follow-up were compared with data of patients lost to follow-up.

\section{Definitions}

Culture-proven EOS was defined as a positive result of one or more bacterial or fungal blood cultures obtained from patients and antimicrobial treatment for at least 5 days. As blood culture has a low sensitivity, especially when limited blood volumes are available, we used a definition of clinical EOS as done in previous similar studies [6, 8, 13, 16, 22]. We defined clinical EOS using established laboratory parameters and persistent clinical presentation $[15,17,18]$ :

1.) either patients show a $\mathrm{CrP} \geq 10 \mathrm{mg} / \mathrm{L}$ in the first 72 $\mathrm{h}$ of life and receive antimicrobial treatment for at least 5 days

2.) or patients with a $\mathrm{CrP} \geq 5 \mathrm{mg} / \mathrm{L}$ have undergone a course of antimicrobial therapy of more than 5 days and present with three or more persistent clinical symptoms as defined below [23]

The following clinical symptoms have been identified in prior research as suggesting an infection and as such have been implemented in German national guidelines $[4,13,23-27]$. These were, in no particular order:

a.) Temperature instability such as fever $>38.0^{\circ} \mathrm{C}$, hypothermia $<36,5^{\circ} \mathrm{C}$, frequent adjustment of the incubator.

b.) Prolonged capillary refill $>2$ sec.

c.) Apnea defined as new or more frequent episodes $>20$ s.

d.) Tachycardia of $>200$ beats/minute.

e.) Poor feeding (increased difficulty in tolerating enteral feeding, i.e. repeated vomiting).

f.) Hypotension presenting with a mean arterial pressure in $\mathrm{mmHg}$ less than gestational age in weeks, or a significant decrease in the patients blood pressure leading to a use of inotropic drugs or pallor. 
g.) Increased oxygen demand with any increase in the amount of oxygen needed to obtain an oxygen saturation of $88-92 \%$ persisting $>60 \mathrm{~min}$.

h.) Acidosis presenting with a base excess $<-10 \mathrm{mEq} / \mathrm{l}$.

In addition we looked at an increase of interleukin-6 laboratory (IL-6, cut-off $\geq 50 \mathrm{pg} / \mathrm{ml}$ ) which, in combination with the above mentioned $\mathrm{CrP}$, has been established over decades as a sensitive marker in the diagnosis of EOS $[15,17,18]$. Given that there is a time lag of $12-24 \mathrm{~h}$ as well as a poor positive predictive accuracy in a single value, increasing serial $\mathrm{CrP}$ values in the same recorded infection period, also past the first 72 $\mathrm{h}$ of life, were taken into the consideration as well [15]. If there were multiple $\mathrm{CrP}$ values at hand, the highest was used in the analyses. Abnormal white blood cell counts (WBC) were recorded using percentiles of VLBW-Infants on the third day of life as published by Obladen et al. [28], with leukocytosis being $>24,5 \mathrm{Gpt} / \mathrm{L}$ (90th percentile) and leukopenia being $<4,8 \mathrm{Gpt} / \mathrm{L}$ (10th percentile). Thrombocytopenia was recorded as a platelet count $<150,000 \mathrm{Gpt} / \mathrm{L}$ and severe thrombocytopenia as a platelet count $<50,000 \mathrm{Gpt} / \mathrm{L}$ [29].

The common denominator of the EOS classification was the incidence of systemic infection in the first $72 \mathrm{~h}$ of life, using established paraclinical parameters and known clinical symptoms evaluated by experienced neonatologists, and leading to antimicrobial therapy for at least 5 days.

The group without EOS consisted of all inborn VLBW infants born during the same time interval but not meeting the criteria of infection within the first $72 \mathrm{~h}$ of life. Patients suffering from infection past $72 \mathrm{~h}$ of life were considered to have LOS [9].

NEC was diagnosed according to the criteria of Bell et al. [30], with clinical and radiological findings meeting the definition of stage II or higher. As in previous studies, patients with NEC (stage II or higher) were considered to be septic as well, as there is a strong association of NEC with infection $[6,8,31]$.

ROP was routinely screened during hospitalization according to the international classification [32]. ROP was considered an adverse outcome, if stage 3 or higher was attained [8]. The incidence and severity of IVH were routinely assessed by cranial ultrasounds after birth, within 2 weeks after birth and prior to discharge, classified according to Papile et al. [33]. Brain injury was interpreted as IVH grade 3 or higher [3, 8, 34]. PVL was assessed during these exams as well, according to the classification of Vries et al. [35], and it was considered as an adverse outcome, if persistent white matter injury (stage 3 or higher) was diagnosed prior to discharge. BPD was characterized as oxygen being supplemented or positive-pressure support being given at 36 weeks postmenstrual age, meeting the criteria of moderate to severe BPD according to the National Institute of Child Health and Development consensus definition [36, 37]. Chorioamnionitis was recorded when a clinical diagnosis according to the hospitals standard operating procedure (maternal fever, persistent elevated temperature, fetal tachycardia >160 min longer than $10 \mathrm{~min}$, maternal leukocytosis, purulent discharge) was confirmed histopathologically or through elevated IL-6 levels in amniocentesis [38].

Z-scores for weight, length, and head circumference were calculated at birth, based on German percentiles by Voigt et al. [39]. The Clinical Risk Index for Babies (CRIB) score, including scores for gestational age, birthweight, maximum and minimum fraction of inspired oxygen and maximum base excess during the first $12 \mathrm{~h}$ of life, was also determined in infants meeting the gestational age criterion of birth below 32 weeks. The criterion of congenital malformation within the CRIB score was irrelevant in our study due to the exclusion criteria [40].

To evaluate the influence of socioeconomic status, parents were subdivided into 4 groups according to information given by parents at admission to hospital as follows: one or more higher educated caregivers with any academic degree (category 4), one or more caregivers having successfully completed a professional training (category 3), caregivers with a high school diploma (category 2) or those without any degree (category 1) $[8,41]$.

\section{Outcome assessment}

All surviving infants were invited to a neurodevelopmental follow-up at 2 years \pm 3 months corrected age using the Bayley Scales of Infant Development (BSID) II in their German translation [42]. The assessment was carried out by experienced neonatologists $(\mathrm{AB})$ as part of routine follow-up examinations in the hospitals neonatology division.

In addition, motor function was assessed according to the modified Gross Motor Function Classification System (GMFCS) to determine cerebral palsy [43].

Neurodevelopmental impairment (NDI) was defined as one or more of the following: Mental Developmental Index (MDI) and/or Psychomotor Developmental Index (PDI) scores lower than 70; presence of cerebral palsy. Furthermore, MDI or PDI scores $<85$ in the absence of cerebral palsy were considered as a secondary outcome $[6,8]$.

\section{Statistical analyses}

Statistical significance for unadjusted comparisons was determined by appropriate tests, including ANOVA, Kruskal-Wallis, $\chi^{2}$ with and without Yates' correction for continuity, Mann-Whitney U, student's t-test including 
the Welch's correction, and Fisher's exact tests. As this was an exploratory analysis, a $p$ value of $<0.05$ was considered significant without correction for multiple testing. Since unevenly distributed risk factors for adverse outcomes are possible confounders for the explored hypotheses, univariate logistic regression analyses were performed to identify such risk factors, also including those identified in the unadjusted comparisons of this study and in previous studies. In the following step, multiple logistic regression analyses were carried out, including variables showing significant differences in the univariate analysis in order to identify independent risk factors for NDI, MDI or PDI results $<70$ or $<85$.

Since multiple regressions done en-bloc for the analysis regarding PDI $<70$ revealed non-interpretable results, owing to close similarities between categories, the infection categories as defined above were individually fed into multiple regressions [44]. All analyses were performed using SPSS Statistics 24 (IBM, New York, USA).

\section{Results}

\section{Study population}

Altogether 405 VLBW infants were born in the study period. Sixty-three were excluded according to the criteria mentioned above, 12 of whom died for reasons not linked to EOS or LOS. For various reasons, including parents moving and/or patients receiving further post-discharge care in other centers closer to their home, 176 patients were lost to follow-up. In the end 166/342 (49\%) remained to be analyzed (Fig. 1). Demographic data of those lost to follow-up were compared to patients with follow-up for validation. Baseline demographic characteristics and clinical data of infants with BSIDII- and those without BSIDII-testing were similar. Only the Apgar 10' score showed a significant difference. However, while being statistically significant, the difference of the average, as well as the median, was less than one score point between both groups (Table 1).

In the following step, demographic data of patients with EOS and without EOS were compared. Risk factors for EOS were - not surprisingly - significantly more frequent in the EOS group. Aside from a higher initial CrP, lower gestational age, and a lower birth weight in the EOS-group, infants in this group were more likely to have a higher CRIB-Score, were more prone to develop $\mathrm{BPD}$, and presented with significantly more of the defined clinical symptoms. Mothers of infants with EOS were more likely to have suffered from chorioamnionitis

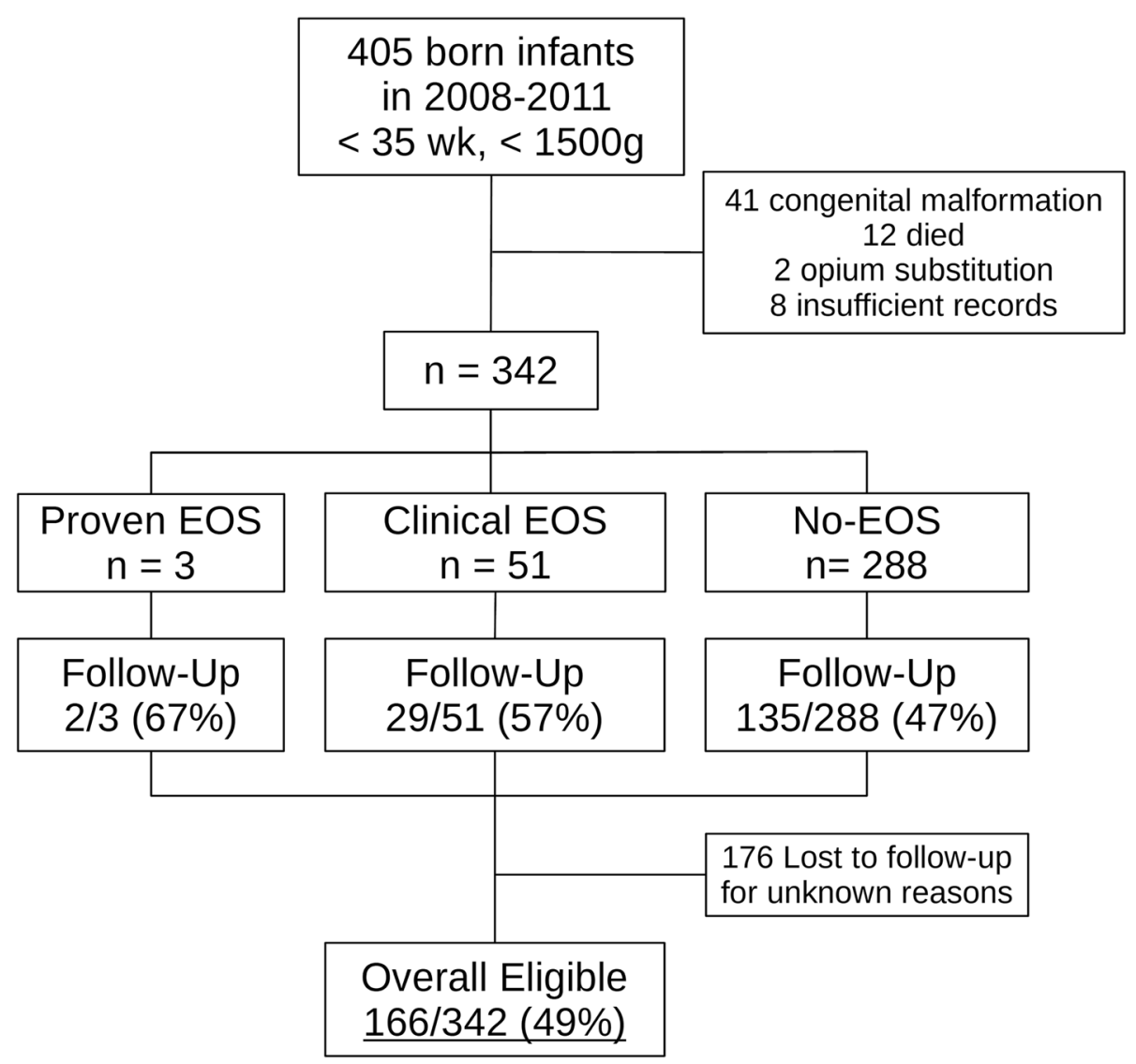

Fig. 1 The graph shows the number of patients from enrollment to follow-up 
Table 1 Demographics

\begin{tabular}{|c|c|c|c|}
\hline & no Follow-Up & Follow-Up & $p$ \\
\hline$N=342 \mathrm{a})$ & $176(51)$ & $166(49)$ & \\
\hline EOS a) & $23(13)$ & $31(19)$ & 0.155 \\
\hline Male, a) & $81(46)$ & $69(42)$ & 0.406 \\
\hline Gestational Age Weeks (wk + d), b) & $29+4( \pm 2+3)$ & $29+1( \pm 2+2)$ & 0.085 \\
\hline Birth weight $g, b$ ) & $1146( \pm 282)$ & $1126( \pm 278)$ & 0.507 \\
\hline Umbilical artery $\mathrm{pH}, \mathrm{b}$ ) & $7.33( \pm 0.30)$ & $7.29( \pm 0.09)$ & 0.124 \\
\hline Apgar $1 ' / 5^{\prime} / 10^{\prime}$, c) & $7 / 8 / 8$ & $7 / 8 / 8$ & $0.250 / 0.144 / 0.022$ \\
\hline Apgar $1^{\prime} / 5^{\prime} / 10^{\prime}$ c) & $(0 ; 6-8 ; 9 / 4 ; 7-8 ; 9 / 6 ; 8-8 ; 10)$ & $(1 ; 6-8 ; 9 / 3 ; 7-8 ; 9 / 5 ; 8-8 ; 10)$ & \\
\hline CRIB-Score, c) & $1(0 / 1-2 / 10)$ & $1(0 / 1-4 / 12)$ & 0.548 \\
\hline Highest Bilirubin, $\mu \mathrm{mol} / \mathrm{l}, \mathrm{b})$ & $145.80( \pm 28.88)$ & $144.77( \pm 33.99)$ & 0.762 \\
\hline Antenatal steroids 1 dose / 2 doses, a) & $87(49) / 53(30)$ & $90(54) / 46(28)$ & $0.478 / 0.624$ \\
\hline Ceaseraen delivery, a) & $162(92)$ & $151(91)$ & 0.589 \\
\hline Patent ductus arteriosus, a) & $35(20)$ & $46(28)$ & 0.408 \\
\hline$B P D, d)$ & $15(9)$ & $12(7)$ & 0.693 \\
\hline Retinopathia neonatorum $\geq 3$, d) & 0 & $2(1)$ & 0.235 \\
\hline Intraventricular haemorrhage $\geq 3$, d) & $1(1)$ & $6(4)$ & 0.061 \\
\hline Periventricular leukomalacia, d) & 0 & $2(1)$ & 0.235 \\
\hline Parental Education Score 1/2 d); 3/4 a) ${ }^{a}$ & $2(1) / 10(6) / 96(55) / 49(28)$ & $1(1) / 9(5) / 93(56) / 57(34)$ & $0.620 / 0.817 / 0.584 / 0.405$ \\
\hline Max CrP 72 h postnatal, c) & $1.04(0 / 0-3.91 / 52.37)$ & $1.04(0 / 0-5.08 / 68.45)$ & 0.748 \\
\hline Leukocytes in first $\left.\mathrm{CBC}, \times 10^{9}, \mathrm{~b}\right)$ & $9.50( \pm 5.86)$ & $9.96( \pm 7.04)$ & 0.512 \\
\hline Last Maternal CrP prior to Birth, c) & $6.82(0 / 1.65-17.25 / 118.3)$ & $7.43(0 / 2.42-21.98$ / 70.77) & 0.330 \\
\hline PROM < 24 h a) $/>24 \mathrm{~h}$ e) $/>1$ week, a) & $55(31) / 15(9) / 29(16)$ & $39(23) / 10(6) / 29(17)$ & $0.108 / 0.412 / 0.699$ \\
\hline Chorioamnionitis, a) & $21(12)$ & $14(8)$ & 0.374 \\
\hline LOS a) & $20(11)$ & $36(22)$ & 0.427 \\
\hline Total sepsis symptoms c) ${ }^{\text {b }}$ & $3(0 / 1-4 / 7)$ & $3(0 / 1-4 / 7)$ & 0.127 \\
\hline
\end{tabular}

a) n (\%), X2-test; b) mean, SD, 1-Way ANOVA; c) median, min, IQR, max; Kruskal-Wallis-Test; d) n (\%), Fisher's exact test;

adisplayed are $\mathrm{n}$ of individual parental education score categories as defined in the methods section

${ }^{b}$ displayed is the median total of clinical symptoms associated with EOS as defined in the methods section

Abbreviations: BPD Bronchopulmonary dysplasia, CBC Complete blood count, CRIB Clinical risk index for babies, EOS Early onset sepsis, LOS Late onset sepsis, PROM Premature rupture of membranes, WBC White blood cell count

and a premature rupture of membranes (PROM) for longer than a week. Furthermore, a statistically significant difference of the Apgar 5' score between EOS and no-EOS groups was noted, which was, as in the prior comparison, less than a score point and was thus considered to be clinically irrelevant (Table 2).

Of the analyzed 166 infants, two had culture-proven sepsis (one caused by a gram-negative pathogen, one by a fungus), 29 had clinical sepsis, and none had NEC (Fig. 1). Of the 29 cases of clinical EOS, five had increased IL-6 serum levels and $13 \mathrm{had}$ abnormal WBCs. Increase in IL-6 and abnormal WBC only coincided in one case. Five patients with clinical sepsis had been exposed to prenatal maternal antibiotics. Thirty-six patients evenly spread among the study groups developed LOS, one even developed meningitis during the hospital stay but had not had EOS before (Table 2). One hundred thirty-five patients did not meet the criteria of EOS.

\section{Outcome assessment}

BSIDII examinations were carried out at a median of 24 months (Range 23 - 31 months) corrected age with no significant difference between the study groups (Table 2). Results of these follow-up examinations are displayed in Table 3 and Fig. 2. MDI values were similar in both study groups, however PDI values were significantly lower in the EOS group. In particular, PDI values $<70$ showed a significant association with infection while PDI values $<85$ only showed a weak association. Moreover, the occurrence of cerebral palsy was significantly higher in the EOS group. Altogether, NDI as defined above was significantly more frequent in the EOS group (Table 3). Two infants of the EOS-group had extremely low MDI or PDI values (Fig. 2). In the case of one bilingual patient, a MDI of 45 was measured due to poor concentration on the test items. 
Table 2 Demographics

\begin{tabular}{|c|c|c|c|}
\hline & No-EOS & EOS & $p$ \\
\hline$N=166$ & 135 & 31 & \\
\hline Male, a) & $54(40)$ & $15(48)$ & 0.514 \\
\hline Gestational Age Weeks $(w k+d), b)$ & $29+2( \pm 2+2)$ & $27+6( \pm 2+0)$ & 0.001 \\
\hline Birth weight $g, b$ ) & $1160.56( \pm 267.62)$ & $974.97( \pm 276.40)$ & 0.001 \\
\hline Umbilical artery $\mathrm{pH}, \mathrm{b}$ ) & $7.30( \pm 0.09)$ & $7.29( \pm 0.08)$ & 0.811 \\
\hline Apgar $\left.1^{\prime} / 5^{\prime} / 10^{\prime}, \mathrm{c}\right)$ & $7 / 8 / 8$ & $7 / 8 / 8$ & $0.125 / 0.041 / 0.057$ \\
\hline Apgar $1^{\prime} / 5^{\prime} / 10^{\prime}$ c) & $(1 ; 6-8 ; 9 / 3 ; 7-8 ; 9 / 5 ; 8-9$ 10) & $(4 ; 6-8 ; 8 / 3 ; 7-8 ; 9 / 3 ; 8-8 ; 9)$ & \\
\hline (RIB-Score, c) & $1(0 / 0-3 / 11)$ & $3(0 / 1.75-5.25 / 12)$ & 0.001 \\
\hline Highest Bilirubin, $\mu \mathrm{mol} / \mathrm{l}, \mathrm{b})$ & $143.85( \pm 23.44)$ & $148.74( \pm 62.31)$ & 0.472 \\
\hline Antenatal steroids 1 dose / 2 doses, a) & $72(53) / 35(26)$ & $18(58) / 11(35)$ & $0.633 / 0.284$ \\
\hline Ceaseraen delivery, a) & $121(90)$ & $30(97)$ & 0.366 \\
\hline Patent ductus arteriosus, d) & $36(27)$ & $10(32)$ & 0.830 \\
\hline$B P D, f)$ & $6(4)$ & $6(19)$ & 0.011 \\
\hline Retinopathia neonatorum $\geq 3$, d) & $2(1)$ & 0 & 0.999 \\
\hline Intraventricular haemorrhage $\geq 3$, d) & $4(3)$ & $2(6)$ & 0.312 \\
\hline Periventricular leukomalacia, d) & $1(1)$ & 1 (3) & 0.340 \\
\hline Parental Education Score 1/2 d); 3/4 a); ${ }^{a}$ ) & $1(1) / 7(5) / 76(56) / 47$ (35) & $0 / 2(6) / 17(55) / 10(32)$ & 0.999 / 0.667 / 0.999 / 0.999 \\
\hline Max CrP 72 h postnatal, c) & $0.62(0 / 0-1.525 / 8.37)$ & 14.36 (5.37 / 9.5-28.07 / 68.45) & 0.000 \\
\hline Leukocytes in first $\left.C B C, \times 10^{9}, b\right)$ & $9.9( \pm 5.71)$ & $10.22( \pm 11.09)$ & 0.824 \\
\hline Leukopenia a) & $9(7)$ & $10(32)$ & 0.000 \\
\hline Leukocytosis d) & $4(3)$ & $3(10)$ & 0.122 \\
\hline Combinded abnormal WBC a) & $13(10)$ & $13(42)$ & 0.000 \\
\hline Thrombocytes in first $\mathrm{CBC}, \times 10^{9}, \mathrm{~b}$ ) & $181.8( \pm 69.7)$ & $173.4( \pm 71.7)$ & 0.547 \\
\hline Thrombocytopenia, a) & $40(30$ & $14(45)$ & 0.146 \\
\hline Severe thrombocytopenia, d) & $3(2)$ & $2(6)$ & 0.234 \\
\hline Last Maternal CrP prior to Birth,c) & $7.21(0 / 2.49-20.41 / 70.77)$ & $8.8(0$ / 2.04-29.16 / 64.93) & 0.541 \\
\hline PROM < 24h / > 24h / > 1 week, d) & $36(27) / 10(7) / 17(13)$ & $3(10) / 0 / 12(39)$ & $0.059 / 0.211 / 0.001$ \\
\hline Chorioamnionitis, d) & $8(6)$ & $6(19)$ & 0.026 \\
\hline LOS d) & $27(20)$ & $9(29)$ & 0.211 \\
\hline Age at BSIDII, month, e) & $24(23-30)$ & $25(24-31)$ & 0.323 \\
\hline Total sepsis symptoms c) ${ }^{b}$ & $3(0 / 2-3.5 / 6)$ & $4(2 / 3-5 / 7)$ & 0.000 \\
\hline
\end{tabular}

a) n (\%), x2-test; b) mean, SD, 1-Way ANOVA; c) median, min, IQR, max; Kruskal-Wallis-Test; d) n (\%), Fisher's exact test; e) median, min max, Mann-Whitney-U-Test

adisplayed are $\mathrm{n}$ of individual parental Education score categories as defined in the methods section

bdisplayed is the median total of clinical symptoms associated with EOS as defined in the methods section

Abbreviations: BPD Bronchopulmonary dysplasia, BSID Bayley Scales of Infant Development II, CBC Complete blood count, CRIB Clinical risk index for babies, EOS

Early onset sepsis, LOS Late onset sepsis, PROM Premature rupture of membranes, WBC White blood cell count

Table 3 Outcome

\begin{tabular}{lllllll}
\hline & EOS $\boldsymbol{n}=\mathbf{3 1}$ & No-EOS $=\mathbf{1 3 5}$ & $\boldsymbol{p}$ & OR & $\mathbf{9 5 \%} \mathbf{C l}$ & $\mathbf{p}$ \\
\hline $\mathrm{NDI}, \mathrm{a})$ & $7(23,58 \%)$ & $11(8,15 \%)$ & 0.015 & 3.288 & $1158-9336$ & 0.025 \\
$\mathrm{PDI}<70, \mathrm{a})$ & $6(19,35 \%)$ & $3(1,65 \%)$ & 0.002 & 10.56 & $2476-45,035$ \\
$\mathrm{MDI}<70, \mathrm{a})$ & $3(9,68 \%)$ & $6(4,44 \%)$ & 0.371 & 2.304 & $0,543-9771$ & 0.001 \\
$\mathrm{PDI}<85, \mathrm{~b})$ & $8(25,81 \%)$ & $18(13,33 \%)$ & 0.088 & 2.261 & $0,879-5818$ & 0.091 \\
$\mathrm{MDI}<85, \mathrm{~b})$ & $7(23,58 \%)$ & $37(27,41 \%)$ & 0.583 & 0.773 & $0,307-1944$ & 0.584 \\
Cerebral Palsy, a) & $4(12,09 \%)$ & $3(1,65 \%)$ & 0.014 & 6.519 & $1379-30,809$ & 0.018 \\
\hline
\end{tabular}

a) $\mathrm{n}, \%$, Fisher's Exact Test; b) n, \%, x2-test

Abbreviations: MDI Motor Development Index, PDI Psychomotor Developmental Index, NDI Neurodevelopmental impairment 


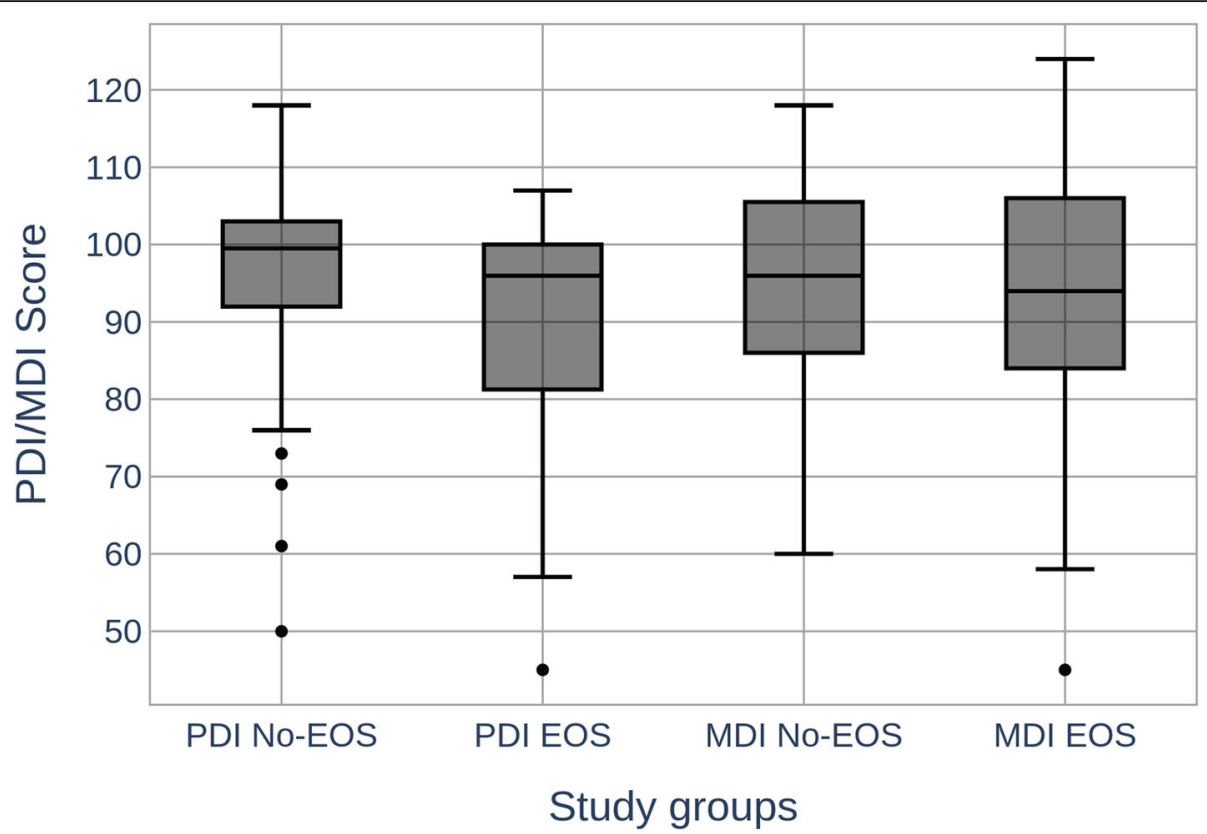

Fig. 2 Displayed are PDI and MDI scores for infants in the EOS and no-EOS groups. Presented are median, quartiles, lower fence and maxima. For MDI Scores in the No-EOS group a minimum is presented. PDI, median, IQR: EOS 96 (80 - 106) No-EOS 99,5 (92 - 103) p=0,029*. MDI: EOS 96 (86 - 106) No-EOS $94(84-106) p=0,771 *$ *Student's T-Test/Welch-Correction

Another infant with a PDI of 45 had been born extremely early and with a severe intrauterine growth retardation (GA $25+6$ weeks, birth weight $445 \mathrm{~g})$.

Logistic regression models showed a few significant risk factors for low MDI or PDI scores and NDI (Tables 4, 5, 6). In the multiple logistic regression analysis, MDI scores $<70$ showed a significant association with the CRIB-Score. Risk factors for PDI $<70$ (in the different analyses) were chorioamnionitis and a high CRIB-Score. No significant individual risk factors for the combined outcome NDI were identified in the multiple logistic regression model. As secondary outcomes MDI scores < 85 showed a weak association with BPD and a significant risk factor for PDI < 85 was IVH (Table 7).

Table 4 Regression analysis PDI $<70$

\begin{tabular}{|c|c|c|c|c|c|c|c|c|}
\hline & Univariable Model & $p$ & $\begin{array}{l}\text { Multivariable Model } \\
\text { a) }\end{array}$ & $p$ & $\begin{array}{l}\text { Multivariable Model } \\
\text { b) }\end{array}$ & $p$ & $\begin{array}{l}\text { Multivariable Model } \\
\text { c) }\end{array}$ & $p$ \\
\hline & OR $(95 \% \mathrm{Cl})$ & & OR $(95 \% \mathrm{Cl})$ & & OR $(95 \% \mathrm{Cl})$ & & OR $(95 \% \mathrm{Cl})$ & \\
\hline EOS & $\begin{array}{l}10.480 \\
(2.457-44.696)\end{array}$ & 0.001 & $5.546(0.568-54.138)$ & 0.141 & & & & \\
\hline Proven EOS & $\begin{array}{l}19.375 \\
(1.108-338.807)\end{array}$ & 0.042 & & & $\begin{array}{l}14.618 \\
(0.179-1193.351)\end{array}$ & 0.232 & & \\
\hline Clinical EOS & $6.550(1.644-26.104)$ & 0.008 & & & & & $2.701(0.306-23.848)$ & 0.371 \\
\hline PROM > 1 week & $4.192(1.052-16.707)$ & 0.042 & $4.176(0.641-27.201)$ & 0.135 & $2.811(0.407-19.390)$ & 0.294 & $4.572(0.681-30.695)$ & 0.118 \\
\hline Chorioamnionitis & $6.591(1.448-29.997)$ & 0.015 & $6.291(0.839-47.175)$ & 0.074 & $7.292(1.029-51.677)$ & 0.047 & $8.724(1.314-57.919)$ & 0.025 \\
\hline Max CrP $72 \mathrm{~h}$ postnatal & $1.048(1.009-1.089)$ & 0.016 & $1.006(0.902-1.099)$ & 0.902 & $1.050(0.982-1.123)$ & 0.153 & $1.022(0.937-1.116)$ & 0.618 \\
\hline CRIB-Score & $1.276(1.042-1.564)$ & 0.019 & $1.311(0.968-1.773)$ & 0.080 & $1.345(1.015-1.782)$ & 0.039 & $1.345(1.000-1.808)$ & 0.050 \\
\hline Highest Bilirubin & $1.014(1.000-1.028)$ & 0.044 & $1.002(0.981-1.023)$ & 0.860 & $0.998(0.978-1.018)$ & 0.815 & $0.999(0.979-1.020)$ & 0.952 \\
\hline $\mathrm{BPD}$ & $4.171(0.764-22.767)$ & 0.099 & & & & & & \\
\hline $\begin{array}{l}\text { Periventricular } \\
\text { leukomalacia }\end{array}$ & $\begin{array}{l}19.375 \\
(1.108-338.807)\end{array}$ & 0.042 & $\begin{array}{l}7.159 \\
(0.246-207.978)\end{array}$ & 0.252 & $\begin{array}{l}12.907 \\
(0.485-343.342)\end{array}$ & 0.127 & $\begin{array}{l}7.735 \\
(0.277-215.872)\end{array}$ & 0.228 \\
\hline
\end{tabular}

Displayed are parameters showing a weak association $(p<0,1)$. Included in the multiple regression models were variables with $p<0,05$ and a) with the combined EOS variables, b) proven EOS and c) only clinical EOS

Abbreviations: CRIB Clinical risk index for babies, EOS Early Onset Infection, PROM Premature rupture of membranes 
Table 5 Regression analysis $\mathrm{MDI}<70$

\begin{tabular}{|c|c|c|c|c|}
\hline & $\begin{array}{l}\text { Univariable Model } \\
\text { OR }(95 \% \mathrm{Cl})\end{array}$ & $p$ & $\begin{array}{l}\text { Multivariable Model } p<0,05 \\
\text { OR }(95 \% \mathrm{Cl})\end{array}$ & $p$ \\
\hline Proven EOS & $19.500(1.115-340.984)$ & 0.042 & $25.559(1.291-506.152)$ & 0.033 \\
\hline CRIB-Score & $1.246(1.026-1.514)$ & 0.027 & $1.269(1.038-1.552)$ & 0.020 \\
\hline BPD & $4.200(0.770-22.922)$ & 0.097 & & \\
\hline
\end{tabular}

Displayed are parameters showing a weak association $(p<0,1)$. Included in the multiple regression models were variables with $p<0,05$

Abbreviations: BPD Bronchopulmonary dysplasia, CRIB Clinical risk index for babies, EOS Early Onset Infection

\section{Discussion}

Infections remain a threat for VLBW-infants, as they are still associated with short- and long-term sequelae and an increased risk of death $[2-4,11]$.

Our findings support the hypothesis that VLBWinfants with EOS in our study cohort had an increased risk for poor neurodevelopmental outcomes at 2 years of age. In fact, the risk was three times higher than in the control group without EOS. It appears that the psychomotoric development was more affected than the mental development. PDI scores were significantly lower in infants who had EOS, and there was an increased number of infants who had cerebral palsy in the EOS group. The differences were not explained by differences in other confounding variables according to the multiple logistic regression results.

Looking at PDI scores, chorioamnionitis as a known maternal precursor for EOS showed a significant negative impact [45]. IVH as an individual affliction of the neonate showed a significant negative impact on the PDI as well. Low MDI scores were weakly associated with BPD in our models. A higher CRIB-Score was related to poorer outcomes in both MDI and PDI.

Our findings concur with previous studies. Fairly recently Ferreira et al. [16] identified clinical sepsis in a Brazilian population as an individual predictor of poorer neurodevelopmental outcomes. In particular the psychomotoric development seemed to be more affected, as well. Research done by Schlapbach et al. [8] showed proven sepsis to be an individual predictor of poorer neurodevelopmental outcomes in infants with EOS and LOS. Before that study, cohorts of Stoll et al. [6] and Bassler et al. [20] had yielded similar results. An association of NDI with culture-proven EOS was shown very recently by Mukhopadhyay et al. [11] on a cohort of extremely preterm infants (gestational age $<27$ weeks) born between 2006 and 2014. Sepsis suspected solely on clinical symptoms, however, did not have a significant association.

As demonstrated here, maternal chorioamnionitis was also shown to affect the PDI in neonates by Klinger et al. [21]. Furthermore, neonatal sepsis has also been established as a risk factor for cerebral palsy $[6,8,46]$.

When comparing our odds ratios to previous studies, the overall risk profile regarding neurodevelopmental impairment caused by EOS seems to have remained fairly similar since the 1990s.

The mechanisms as to how neonatal sepsis causes brain injury, however, notably without direct CNS involvement such as intracranial hemorrhage or meningitis, are still unknown. A study using MRI imaging has shown white matter injury in patients with recurrent postnatal infections [47]. In general a higher vulnerability to injury because of the immaturity of the developing brain is postulated $[7,9]$. Several causative mechanisms have been suggested, including cytokines being transported actively across the intact blood brain barrier, a potential mechanism through the activation of the hypothalamic-pituitary-adrenal-axis, a pathway across the blood brain barrier at circumventricular organs, and a 'leak' across an intact blood brain barrier with altered permeability through inflammation or cytokines being produced by cells infiltrating the CNS [5, 7]. Although it appears that the inflammatory cytokine response precedes and contributes to brain damage, there are additional risks of brain damage during EOS through

Table 6 Regression Analysis NDI

\begin{tabular}{lllll}
\hline & $\begin{array}{l}\text { Univariable Model } \\
\text { OR }(\mathbf{9 5 \%} \text { Cl) }\end{array}$ & $\boldsymbol{p}$ & \multicolumn{1}{l}{$\begin{array}{l}\text { Multivariable Model } \boldsymbol{p}<\mathbf{0 , 0 5} \\
\text { OR (95\% Cl) }\end{array}$} \\
\hline EOS & $3.29(1.16-9.34)$ & 0.025 & $2.652(0.804-8.743)$ & 0.109 \\
Clinical EOS & $2.58(0.88-7.554)$ & 0.083 & & \\
CRIB-Score & $1.22(1.04-1.42)$ & 0.012 & $1.121(0.928-1.353)$ & 0.236 \\
Highest Bilirubin & $1.01(1.00-1.03)$ & 0.046 & $1.007(0.992-1.022)$ & 0.377 \\
Intraventricular haemorrhage $\geq 3$ & $9.67(1.79-52.19)$ & 0.008 & $4.309(0.535-34.726)$ & 0.170 \\
\hline
\end{tabular}

Displayed are parameters showing a weak association $(p<0,1)$. Included in the multiple regression models were variables with $p<0,05$ Abbreviations: CRIB Clinical risk index for babies, EOS Early Onset Infection 
Table 7 Secondary Outcome

\begin{tabular}{|c|c|c|c|c|}
\hline & $\begin{array}{l}\text { Univariable Model } \\
\text { OR }(95 \% \mathrm{Cl})\end{array}$ & $p$ & $\begin{array}{l}\text { Multivariable Model } p<0,05 \\
\text { OR }(95 \% \mathrm{Cl})\end{array}$ & $p$ \\
\hline \multicolumn{5}{|l|}{ Regression analysis $\mathrm{MDI}<85$} \\
\hline Gestational Age & $0.973(0.951-0.996)$ & 0.022 & $0.994(0.960-1.030)$ & 0.756 \\
\hline Birthweight & $0.998(0.997-0.999)$ & 0.002 & $0.999(0.997-1.002)$ & 0.578 \\
\hline Male Gender & $2.023(0.966-4.237)$ & 0.062 & & \\
\hline CRIB-Score & $1.282(1.124-1.462)$ & 0.000 & $1.145(0.930-1.411)$ & 0.202 \\
\hline BPD & $6.556(1.865-23.040)$ & 0.003 & $3.392(0.853-13.485)$ & 0.083 \\
\hline \multicolumn{5}{|l|}{ Regression analysis PDI $<85$} \\
\hline EOS & $2.242(0 . .871-5769)$ & 0.094 & & \\
\hline PROM > 1 week & $2.497(0.962-6.480)$ & 0.060 & & \\
\hline Gestational Age & $0.955(0.926-0.984)$ & 0.003 & $0.983(0.938-1.031)$ & 0.480 \\
\hline Birth weight & $0.997(0.996-0.999)$ & 0.002 & $1.00(0.997-1.003)$ & 0.952 \\
\hline Apgar 10' & $0.548(0.305-0.986)$ & 0.045 & $0.647(0.289-1.45)$ & 0.290 \\
\hline CRIB-Score & $1.352(1.168-1.564)$ & 0.000 & $1.128(0.863-1.475)$ & 0.377 \\
\hline BPD & $6.650(1.953-22.645)$ & 0.002 & $3.408(0.775-14.994)$ & 0.105 \\
\hline Intraventricular haemorrhage $\geq 3$ & $32.857(3.657-295.224)$ & 0.002 & $18.137(1.676-196.218)$ & 0.017 \\
\hline LOS & $3.939(0.798-19.453)$ & 0.092 & & \\
\hline
\end{tabular}

Displayed are parameters showing a weak association $(p<0,1)$. Included in the multiple regression models were variables with $p<0,05$ Abbreviations: BPD Bronchopulmonary dysplasia, CRIB Clinical risk index for babies, EOS Early Onset Infection, PROM premature rupture of membranes

haemodynamic instability and respiratory disease as well. Hypoxaemia and pathological alterations in cerebral blood flow may pose a risk for neurodevelopmental outcome by themselves $[6,7,20]$. BPD as an individual risk factor of NDI is associated with cytokine release from the lungs, sepsis, and with an increased exposure to ventilation and oxygen in infants with EOS [21].

Avoiding EOS continues to be challenging in the prevention of NDI. Even optimized therapy protocols seem to have resulted, if at all, in only a minor reduction in NDI in affected patients. Reducing EOS itself may be most effective in increasing the proportion of infants surviving without NDI [48]. Identifying EOS remains a major obstacle in the prevention of NDI, with the gold standard still being proof by culture. A study by Mikhael et al. [22] of 1593 neonates $\geq 23$ weeks' gestation, who underwent an EOS evaluation $\leq 72 \mathrm{~h}$ postnatal due to clinical signs and an elevated risk for EOS, found only 9 cases $(0.56 \%)$ of proven EOS. The potentially large difference between incidence of culture proven EOS, and patients presenting with clinical symptoms of an infection, was demonstrated in other studies as well, most recently by Mukhopady et al. [8, 11, 16]. In their study of the impact of EOS and antibiotic use on patients with an extremely low birth weight (< $1000 \mathrm{~g}$ ) culture-confirmed EOS occurred in $2.3 \%$ of the patients, even though $48.6 \%$ of them received antibiotic treatment for more than 5 days.
Similar differences between incidence rates of clinically diagnosed sepsis and culture proven sepsis can be seen in the German 'Neonatalerhebung' (a registry of neonatal births in all German perinatal centers), as well as in studies carried out in developing countries $[10,16,49]$. A discussion as to how to define sepsis in different pediatric patient collectives was led in the past years $[14,50]$. Without a universally accepted sepsis definition, especially for neonatal patients, researchers have made attempts similar to ours using clinical and paraclinical parameters to define clinical EOS in their studies $[6,8,11]$. Fairly recently, following the Sepsis-3 consensus definition in adults, promising steps have been made to enter definitions of organ failure using scoring systems to improve identification of sepsis $[14,51-53]$. However, the wide range of pediatric patients, and even of neonates, remains an obstacle to these efforts. Wynn et al. [53] introduced a neonatal sequential organ failure score (nSOFA) including a risk calculator for LOS patients. They aim to validate and possibly adapt the nSOFA for EOS patients in future multi-center research, too. In fact, recent research of the group has shown very promising results validating the nSOFA for LOS patients [54].

Furthermore, a priori risk stratification scores have been established, which have lead to the Kaiser EOS calculator, using maternal as well as patients' characteristics (e.g. maternal GBS status, PROM, gestational age and clinical status) to allow an identification of patients at a higher risk of EOS $[4,12,19,26]$. With a more accurate 
estimation of neonates at risk, clinicians are able to monitor high risk patients more closely, and reduce the number of patients being treated unnecessarily with antibiotics, thus reducing the risks of antibiotic treatment itself $[4,26,55]$. This risk reduction is directly linked to short and long term neonatal outcomes such as, among others, increased risk of NEC, fungal infections, and death, or alterations to the microbiome, allergies, and autoimmune diseases [4, 55]. In fact, Mukhopadhyay et al. [11] reported that antibiotic treatment for 5 days or more, in the absence of culture proven sepsis, by itself showed a trend towards adverse neurodevelopmental outcome, which might be explained by a portion of these infants actually having sepsis, but negative cultures. Highly sensitive and specific blood biomarkers for sepsis were unfortunately not considered.

A few limitations of this study need to be addressed. The single center design and the focus on EOS of our study avoided inter-center variations, but limited the available sample size. Only two cases of culture-proven EOS were available in the study group. However, with known incidence rates and research into the sensitivity of blood culture in neonatal care, our reported numbers fall into the expected range [12, 22]. Additionally, in five cases prenatal maternal antibiotics may have caused sterile blood cultures in clinical EOS patients. Some potentially confounding conditions, which have shown an impact on NDI in previous studies, (i.e. ROP, NEC, IVH), were too rare in our cohort to be statistically analyzed $[1,6,8]$. Owing to the retrospective design, not all important parameters were measured in all infants, and some, such as IL-6, were only sparsely measured at all. Other findings associated with EOS, such as severe thrombocytopenia, were too rare to show significant differences between the study groups. This was to be expected due to, first of all, a wide range of morbidities causing early onset thrombocytopenia, and, second of all, as shown in prior research, only a small increase in the odds of EOS with low platelet counts [56]. As discussed above, the maternal GBS status would have further improved the stratification of our study groups, but was unfortunately not accessible to us, since prenatal GBS cultures are not part of standard care in Germany [19]. The surrounding sociogeographic structures of our perinatal center led to a proportion of patients being lost to follow-up. Especially in the first year of our study, there was a high proportion lost to follow-up as followup exams only became mandatory in Germany in 2009. The rates of infection and basic demographic data were very similar in both groups with and without follow-up, indicating that our study group is representative (Table 1). Demographic differences in the comparison between the EOS and No-EOS groups can be explained on the one hand by known risk factors of EOS, such as prematurity, low-birth weight or chorioamnionitis, and on the other by the differentiation of the group itself, i.e. CrP and WBC values. The difference in CRIB-scores may be explained by the overlap of criteria included in the score with the above mentioned parameters. Such differences were to be expected [9].

The large extent of clinical and paraclinical factors linked to NDI in premature infants by themselves may impede determining the individual cause of an adverse outcome, especially as such factors may be linked to EOS as well. In our study such parameters (among others lower birth weight, lower gestational age, BPD, antibiotic treatment) showed significant differences between the study groups. In fact, the CRIB score was, not surprisingly, identified as a risk factor in our regression analyses, since it employs a combination of the above mentioned parameters. Additionally physiologic clinical alterations in the neonatal period may be mistaken for pathological sepsis symptoms. Therefore studies into EOS and LOS using clinical definitions need to be interpreted with caution, since clinical presentations may have a low specificity. However, as Stoll et al. [12] recently reported, in their study cohort nearly all infected infants had signs of instability within $72 \mathrm{~h}$ after birth and the clinical diagnosis remains essential in the early detection of infection $[19,26,27]$. We aimed to overcome these weaknesses through a combination of clinical and paraclinical findings, as was shown in prior research to improve specificity $[4,13,15,19,23,24,26]$. The correlation between significant differences in EOS and noEOS groups in the occurrence of clinical symptoms and blood biomarkers, as well as the consistency of our findings with prior research, could be interpreted in favor of our clinical EOS definition and thus pointing to EOS as cause of NDI (Table 2).

The differences in Apgar scores between demographic groups of less than one score point have no predictive value for neurodevelopmental outcomes, making a bias unlikely [57] (Tables 1 and 2). The limited sample size and low numbers in specific adverse outcomes limited the ability to perform multivariate analyses, as seen to some extent in previous studies as well $[8,16]$. Therefore, odds ratios are to be interpreted with caution as, here, they tended to have rather wide confidence intervals [44]. In addition, like many other studies evaluating neurodevelopmental outcomes, our study is based on outcomes determined in a single assessment at the corrected age of 2 years [58]. Later cognitive and neuromotor outcomes, such as specific learning difficulties or milder motor dysfunctions in light of our tendency for poorer PDI outcomes, are not part of the study because such data is not available $[8,59,60]$. Another limitation to our study, as well as others, is death after discharge from hospital not being recorded as a competing 
outcome $[6,8,16]$. However, most deaths in EOS patients occur in the postnatal period in hospital and no deaths linked to EOS were recorded in our patients [12].

Strengths of our study include a focused assessment into the effects of EOS, and an EOS definition with improved sensitivity and specificity by using established blood infection markers. LOS, as mentioned above, was distributed evenly among the study groups, making a bias herein unlikely. Since this is a single-center study, our study cohort was assessed by the same definitions and treated according to the same standard operating procedures. The BSID-II exam was carried out by a single experienced neonatologist $(\mathrm{AB})$.

\section{Conclusion}

A significant association of EOS with an increased risk of poor neurodevelopmental outcomes in VLBW-infants was demonstrated. Measures for earlier detection, prevention, and improved treatment standards have shown a reduction in infections, while the risk of neurodevelopmental impairment in patients suffering from EOS seems to be fairly constant. Hence, while there has been improvement, neurodevelopmental impairment caused by EOS and the pathogenesis need to stay in focus in future research to further improve understanding and, consequently, therapy. Perinatal standard operating procedures for mother and child need to be focused on prevention, and earlier detection for a quick therapeutic reaction.

\section{Abbreviations}

BPD: Bronchopulmonary dysplasia; BSIDII: Bayley Scale of Infant Development II; CBC: Complete blood count; CNS: Central nervous system; CRIBScore: Clinical Risk Index for Babies; EOS: Early-onset-sepsis; GBS: Group-BStreptococcus; GMFCS: Gross Motor Function Classification System; IL6: Interleukin-6; IVH: Intraventricular hemorrhage; LOS: Late-onset-sepsis; MDI: Mental Developmental Index; NDI: Neurodevelopmental impairment; NEC: Necrotizing enterocolitis; NICU: Neonatal intensive care unit; PDA: Patent ductus arteriosus; PDI: Psychomotor Developmental Index; PROM: Premature rupture of membranes; ROP: Retinopathy of prematurity; SIRS: Systemic inflammatory response syndrome; VLBW: Very low birth weight (<1500 g); WBC: White blood cell count

\section{Acknowledgments}

We would like to thank Emma Martín Rodríguez, Dirk Ortgies (both IRYCIS Madrid), Bruce R. Whittlesey (Texas Tech University) and Anke McLeod (Department of Nuclear Medicine, University Hospital Leipzig) for their careful review of the manuscript.

\section{Authors' contributions}

Division of Neonatology, University Children's Hospital Leipzig, Germany. T.O.: Writing - Original Draft, Review \& Editing, Conceptualiziation, Data Curation, Formal analysis, Methodology, Validation, Visualization, Project administration. A.B.: Data Curation, Investigation, Resources. D.Z.: Investigation. U.T.: Supervision, Writing - Review \& Editing, Conceptualization, Methodology, Resources, Project administration. Department of Nuclear Medicine, University of Leipzig, Germany. M.R.: Data Curation, Formal analysis, Software, Validation. The authors read and approved the final manuscript.

\section{Funding}

Open Access funding enabled and organized by Projekt DEAL.

\section{Availability of data and materials}

The datasets used and/or analyzed during the current study are available from the corresponding author upon reasonable request. (Correspondence to tjark.ortgies@gmx.de).

\section{Declarations}

\section{Ethics approval and consent to participate}

The research was carried out in adherence to ethical standards required by the University Hospital Leipzig in accordance with German state law (Sächsisches Krankenhausgesetz - SächsKHG, § 34) and the principles defined through the Declaration of Helsinki. In accordance with the above mentioned law and institutional requirements, individual ethical review and approval was not required for the study on human participants, as no direct testing on human subjects was done and only existing data was retrieved from medical records. Written informed consent from the patients' legal guardian/next of kin to use data in retrospective analyses, in adherence to data protection law, is attained by admission to the hospital. ( $\$ 34$ SächsKHG \$203 Strafgesetzbuch).

\section{Competing interests}

The authors acknowledge support from the German Research Foundation (DFG) and Universität Leipzig within the program of Projekt DEAL. The contents of the manuscript are solely the responsibility of the authors. The funding source was not involved in the analyses or interpretation of data. We, the authors of this article, certify that there is neither a conflict of interest with any financial organization nor a non-financial interest in the subject matter discussed in this manuscript.

\section{Author details}

'Division of Neonatology, University Children's Hospital Leipzig, Liebigstraße 20, 04103 Leipzig, Germany. ${ }^{2}$ Department of Nuclear Medicine, University Hospital Leipzig, Liebigstraße 18, 04103 Leipzig, Germany.

Received: 1 January 2021 Accepted: 24 May 2021

Published online: 25 June 2021

\section{References}

1. Stoll BJ, Hansen NI, Bell EF, Shankaran S, Laptook AR, Walsh MC, et al. Neonatal outcomes of extremely preterm infants from the NICHD neonatal research network. Pediatrics. 2010;126(3):443-56. https://doi.org/10.1542/ peds.2009-2959

2. Saigal S, Doyle LW. An overview of mortality and sequelae of preterm birth from infancy to adulthood. Lancet. 2008;371(9608):261-9. https://doi.org/1 0.1016/S0140-6736(08)60136-1.

3. Fanaroff AA, Stoll BJ, Wright LL, Carlo WA, Ehrenkranz RA, Stark AR, et al. Trends in neonatal morbidity and mortality for very low birthweight infants. Am J Obstet Gynecol. 2007;196:147.e1-8. https://doi.org/10.1016/j.ajog.2006. 09.014.

4. Puopolo KM, Benitz WE, Zaoutis TE. Management of Neonates Born at $\leq 34$ 6/7 weeks' gestation with suspected or proven early-onset bacterial Sepsis. Pediatrics. 2018;142(6):e20182896. https://doi.org/10.1542/peds.2018-2896.

5. Polin RA. Systemic infection and brain injury in the preterm infant. J Pediatr. 2008;84(3):188-91. https://doi.org/10.2223/JPED.1784

6. Stoll BJ. Neurodevelopmental and growth impairment among extremely low-birth-weight infants with neonatal infection. JAMA. 2004;292(19):235765. https://doi.org/10.1001/jama.292.19.2357.

7. Edwards $A D$, Tan S. Perinatal infections, prematurity and brain injury. Curr Opin Pediatr. 2006;18(2):119-24. https://doi.org/10.1097/01.mop.0000193290. 02270.30 .

8. Schlapbach $L$, Aebischer M, Adams M, Natalucci G, Bonhoeffer J, Latzin P, et al. Impact of Sepsis on neurodevelopmental outcome in a Swiss National Cohort of extremely premature infants. Pediatrics. 2011;128(2):e348-57. https://doi.org/10.1542/peds.2010-3338.

9. Simonsen KA, Anderson-Berry AL, Delair SF, Davies HD. Early-onset neonatal Sepsis. Clin Microbiol Rev. 2014;27(1):21-47. https://doi.org/10.1128/CMR. 00031-13.

10. Zea-Vera A, Ochoa TJ. Challenges in the diagnosis and management of neonatal sepsis. J Trop Pediatr. 2015;61(1):1-13. https://doi.org/10.1093/ tropej/fmu079. 
11. Mukhopadhyay S, Puopolo KM, Hansen NI, Lorch SA, DeMauro SB, Greenberg RG, et al. Impact of Early-Onset Sepsis and Antibiotic Use on Death or Survival with Neurodevelopmental Impairment at 2 Years of Age among Extremely Preterm Infants. J Pediatr. 2020;221:39-46.e5. https://doi. org/10.1016/j.jpeds.2020.02.038

12. Stoll BJ, Puopolo KM, Hansen NI, Sánchez PJ, Bell EF, Carlo WA, et al. Eunice Kennedy Shriver National Institute of Child Health and Human Development Neonatal Research Network Early-onset neonatal Sepsis 2015 to 2017, the rise of Escherichia coli, and the need for novel prevention strategies. JAMA Pediatr. 2020;174(7):1-12. https://doi.org/10.1001/jama pediatrics.2020.0593.

13. Ohlin A, Björkqvist M, Montgomery SM, Schollin J. Clinical signs and CRP values associated with blood culture results in neonates evaluated for suspected sepsis. Acta Paediatr. 2010;99(11):1635-40. https://doi.org/1 0.1111/j.1651-2227.2010.01913.x.

14. Wynn JL. Defining neonatal sepsis. Curr Opin Pediatr. 2016;28(2):135-40. https://doi.org/10.1097/MOP.0000000000000315.

15. Ganesan P, Shanmugam P, Sattar SBA, Shankar SL. Evaluation of IL-6, CRP and hs-CRP as early markers of neonatal sepsis. J Clin Diagn Res. 2016;10: 13-7. https://doi.org/10.7860/JCDR/2016/19214.7764.

16. Ferreira RC, Mello RR, Silva KS. Neonatal sepsis as a risk factor for neurodevelopmental changes in preterm infants with very low birth weight $\pi$ - a sepse neonatal Como fator de Risco Para alteração no neurodesenvolvimento em prematuros de muito baixo peso ao nascer. J Pediatr. 2014;90(3):293-9. Available at: http://www.scielo.br/scielo. php?script=sci_arttext\&pid=S0021-75572014000300293. https://doi.org/10.1 016/j.jped.2013.09.006

17. Franz AR. Measurement of interleukin 8 in combination with C-reactive protein reduced unnecessary antibiotic therapy in newborn infants: a multicenter, randomized, controlled trial. Pediatrics. 2004;114(1):1-8. https:// doi.org/10.1542/peds.114.1.1.

18. Mathers NJ, Pohlandt F. Diagnostic audit of C-reactive protein in neonatal infection. Eur J Pediatr. 1987;146(2):147-51. https://doi.org/10.1007/ BF02343221.

19. Puopolo KM, Lynfield R, Cummings JJ. Management of infants at risk for group B streptococcal disease. Pediatrics. 2019;144(2):e20191881. https://doi. org/10.1542/peds.2019-1881.

20. Bassler D, Stoll BJ, Schmidt B, Asztalos EV, Roberts RS, Robertson CMT, et al. Using a count of neonatal morbidities to predict poor outcome in extremely low birth weight infants: added role of neonatal infection. Pediatrics. 2009;123(1):313-8. https://doi.org/10.1542/peds.2008-0377.

21. Klinger G, Levy I, Sirota L, Boyko V, Lerner-Geva L, Reichman B. Outcome of early-onset Sepsis in a National Cohort of very low birth weight infants. Pediatrics. 2010;125(4):e736-40. https://doi.org/10.1542/peds.2009-2017.

22. Mikhael M, Brown LS, Rosenfeld CR. Serial neutrophil values facilitate predicting the absence of neonatal early-onset sepsis. Journal of Pediatrics. 2014;164:522-528.e3. https://doi.org/10.1016/j.jpeds.2013.10.080.

23. Modi N, Doré CJ, Saraswatula A, Richards M, Bamford KB, Coello R, et al. A case definition for national and international neonatal bloodstream infection surveillance. Arch Dis Child Fetal Neonatal Ed. 2009;94(1):8-13. https://doi.org/10.1136/adc.2007.126458.

24. Zemlin M, Berger A, Franz A, Gille C, Härtel C, Küster H, et al. Bakterielle Infektionen bei Neugeborenen. Leitlinie der GNPI, DGPI, DGKJ und DGGG. (S2k-Level, AWMF-Leitlinien-Register-Nr. 024/008, April 2018). Zeitschrift für Geburtshilfe und Neonatologie. 2019;223:130-44. https://doi.org/10.1055/a0756-7338.

25. Surveillance von nosokomialen Infektionen, multiresistenten Erregern und Antibiotika-Anwendungen bei Frühgeborenen mit einem Geburtsgewicht unter 1.500g. (2020): 1-43. Available at: https://www.nrz-hygiene.de/surveilla nce/kiss/neo-kiss/

26. Kuzniewicz MW, Puopolo KM, Fischer A, Walsh EM, Li S, Newman TB, et al. A Quantitative, Risk-Based Approach to the Management of Neonatal EarlyOnset Sepsis. JAMA Pediatr. 2017;171(4):365-71. https://doi.org/10.1001/ja mapediatrics.2016.4678.

27. Ussat M, Vogtmann C, Gebauer C, Pulzer F, Thome U, Knüpfer M. The role of elevated central-peripheral temperature difference in early detection of lateonset sepsis in preterm infants. Early Hum Dev. 2015;91(12):677-81. https:// doi.org/10.1016/j.earlhumdev.2015.09.007.

28. Obladen M, Diepold K, Maier RF. Venous and arterial hematologic profiles of very low birth weight infants. Pediatrics. 2000;106(4):707-11. https://doi. org/10.1542/peds.106.4.707.
29. Roberts I, Stanworth S, Murray NA. Thrombocytopenia in the neonate. Blood Rev. 2008;22(4):173-86. https://doi.org/10.1016/j.blre.2008.03.004.

30. Bell MJ, Ternberg JL, Feigin RD, Keating JP, Marshall R, Barton L, et al. Neonatal necrotizing enterocolitis. Therapeutic decisions based upon clinical staging. Ann Surg. 1978;187:1-7. https://doi.org/10.1097/00000658-1 97801000-00001.

31. Willoughby RE, Pickering LK. Necrotizing Enterocolitis and infection. Clin Perinatol. 1994;21(2):307-15. https://doi.org/10.1016/S0095-5108(18)30347-6.

32. ICROP. The International Classification of Retinopathy of Prematurity Revisited. Arch Ophthalmol. 2005;123:991. https://doi.org/10.1001/a rchopht.123.7.991.

33. Papile L-A, Burstein J, Burstein R, Koffler H. Incidence and evolution of subependymal and intraventricular hemorrhage: a study of infants with birth weights less than 1,500 gm. J Pediatr. 1978;92(4):529-34. https://doi. org/10.1016/S0022-3476(78)80282-0.

34. Papile L-A, Munsick-Bruno G, Schaefer A. Relationship of cerebral intraventricular hemorrhage and early childhood neurologic handicaps. J Pediatr. 1983;103(2):273-7. https://doi.org/10.1016/S0022-3476(83)80366-7.

35. de Vries LS, Eken P, Dubowitz LMS. The spectrum of leukomalacia using cranial ultrasound. Behav Brain Res. 1992;49(1):1-6. https://doi.org/10.1016/ S0166-4328(05)80189-5.

36. Walsh MC, Wilson-Costello D, Zadell A, Newman N, Fanaroff A. Safety, reliability, and validity of a physiologic definition of bronchopulmonary dysplasia. J Perinatol. 2003;23(6):451-6. https://doi.org/10.1038/sj.jp.7210963.

37. Walsh MC. Impact of a physiologic definition on Bronchopulmonary dysplasia rates. Pediatrics. 2004;114(5):1305-11. https://doi.org/10.1542/peds.2004-0204.

38. Thomas W, Speer CP. Chorioamnionitis: important risk factor or innocent bystander for neonatal outcome? Neonatology. 2011;99(3):177-87. https:// doi.org/10.1159/000320170.

39. Voigt M, Schneider K, Jährig K. Analyse des Geburtengutes des Jahrgangs 1992 der Bundesrepublik Deutschland. Geburtshilfe Frauenheilkd. 1996; 56(10):550-8. https://doi.org/10.1055/s-2007-1023283.

40. The International Neonatal Network. The CRIB (clinical risk index for babies) score: a tool for assessing initial neonatal risk and comparing performance of neonatal intensive care units. Lancet. 1993;342(8865):193-8. https://doi. org/10.1016/0140-6736(93)92296-6.

41. Roberts E, Bornstein MH, Slater AM, Barrett J. Early cognitive development and parental education. Infant Child Dev. 1999;8(1):49-62. https://doi.org/1 0.1002/(SICI)1522-7219(199903)8:1<49::AID-ICD188>3.0.CO;2-1.

42. Bayley N. Manual for the Bayley scales of infant development. 2nd ed. San Antonio, TX: Psychological Corporation; 1993.

43. Palisano R, Rosenbaum P, Walter S, Russell D, Wood E, Galuppi B. Development and reliability of a system to classify gross motor function in children with cerebral palsy. Dev Med Child Neurol. 2008;39(4):214-23. https://doi.org/10.1111/j.1469-8749.1997.tb07414.x.

44. Ranganathan P, Pramesh CS, Aggarwal R. Common pitfalls in statistical analysis: logistic regression. Perspect Clin Res. 2017:8(3):148-51. https://doi. org/10.4103/picr.PICR_87_17.

45. Hornik CP, Fort P, Clark RH, Watt K, Benjamin DK, Smith PB, et al. Early and late onset sepsis in very-low-birth-weight infants from a large group of neonatal intensive care units. Early Hum Dev. 2012;88:S69-74. https://doi. org/10.1016/S0378-3782(12)70019-1.

46. Wheater M, Rennie JM. Perinatal infection is an important risk factor for cerebral palsy in very-low-birthweight infants. Dev Med Child Neurol. 2000; 42(6):364-7. https://doi.org/10.1017/S0012162200000670.

47. Glass HC, Bonifacio SL, Chau V, Glidden D, Poskitt K, Barkovich AJ, et al. Recurrent postnatal infections are associated with progressive white matter injury in premature infants. Pediatrics. 2008;122(2):299-305. https://doi.org/1 0.1542/peds.2007-2184.

48. Shane AL, Stoll BJ. Neonatal sepsis: Progress towards improved outcomes. J Infect. 2014;68:S24-32. https://doi.org/10.1016/j.jinf.2013.09.011.

49. Institut für angewandte Qualitätsförderung und Forschung im Gesundheitswesen GmbH. NEO - Neonatalerhebung 2014. (2015). Available at: https://sqg.de/downloads/Bundesauswertungen/2014/bu_Gesamt_ NEO_2014.pdf

50. Molloy EJ, Wynn JL, Bliss J, Koenig JM, Keij FM, McGovern M, et al. Neonatal sepsis: need for consensus definition, collaboration and core outcomes. Pediatr Res. 2020;88(1):2-4. https://doi.org/10.1038/s41390-020-0850-5.

51. Schlapbach LJ, Straney L, Bellomo R, MacLaren G, Pilcher D. Prognostic accuracy of age-adapted SOFA, SIRS, PELOD-2, and qSOFA for in-hospital mortality among children with suspected infection admitted to the 
intensive care unit. Intensive Care Med. 2018;44(2):179-88. https://doi.org/1 0.1007/s00134-017-5021-8.

52. Singer M, Deutschman CS, Seymour CW, Shankar-Hari M, Annane D, Bauer $M$, et al. The Third International Consensus Definitions for Sepsis and Septic Shock (Sepsis-3). JAMA. 2016;315:801. https://doi.org/10.1001/ja ma.2016.0287.

53. Wynn JL, Polin RA. A neonatal sequential organ failure assessment score predicts mortality to late-onset sepsis in preterm very low birth weight infants. Pediatr Res. 2020;88(1):85-90. https://doi.org/10.1038/s41390-0190517-2.

54. Fleiss N, Coggins SA, Lewis AN, Zeigler A, Cooksey KE, Walker LA, et al. Evaluation of the neonatal sequential organ failure assessment and mortality risk in preterm infants with late-onset infection. JAMA Netw Open. 2021;4(2):e2036518. https://doi.org/10.1001/jamanetworkopen.2020.36518.

55. Achten NB, Klingenberg C, Benitz WE, Stocker M, Schlapbach LJ, Giannoni E, et al. Association of use of the neonatal early-onset Sepsis calculator with reduction in antibiotic therapy and safety: a systematic review and metaanalysis. JAMA Pediatr. 2019;173(11):1032-40. https://doi.org/10.1001/jama pediatrics.2019.2825.

56. Hornik CP, Benjamin DK, Becker KC, Benjamin DK, Li J, Clark RH, et al. Use of the complete blood cell count in early-onset neonatal sepsis. Pediatr Infect Dis J. 2012:31(8):799-802. https://doi.org/10.1097/INF.0b013e318256905c.

57. Jaffe S. The Apgar Score. Pediatrics. 2015;136(4):819-22. https://doi.org/10.1 542/peds.2015-2651.

58. Cai S, Thompson DK, Anderson PJ, Yang JY. Outcomes of very preterm infants with neonatal Sepsis : a systematic review and meta-analysis; 2019.

59. Hack M. Poor predictive validity of the Bayley scales of infant development for cognitive function of extremely low birth weight children at school age. Pediatrics. 2005;1 16(2):333-41. https://doi.org/10.1542/peds.2005-0173.

60. Marlow N, Wolke D, Bracewell MA, Samara M. Neurologic and developmental disability at six years of age after extremely preterm birth. N Engl J Med. 2005;352(1):9-19. https://doi.org/10.1056/NEJMoa041367.

\section{Publisher's Note}

Springer Nature remains neutral with regard to jurisdictional claims in published maps and institutional affiliations.

Ready to submit your research? Choose BMC and benefit from:

- fast, convenient online submission

- thorough peer review by experienced researchers in your field

- rapid publication on acceptance

- support for research data, including large and complex data types

- gold Open Access which fosters wider collaboration and increased citations

- maximum visibility for your research: over $100 \mathrm{M}$ website views per year

At $\mathrm{BMC}$, research is always in progress.

Learn more biomedcentral.com/submissions 